MATHEMATICS OF COMPUTATION

Volume 67, Number 221, January 1998, Pages 439-440

S $0025-5718(98) 00881-3$

\title{
A CONJECTURE OF ERDÖS ON 3-POWERFUL NUMBERS
}

\author{
J. H. E. COHN
}

\begin{abstract}
Erdös conjectured that the Diophantine equation $x+y=z$ has infinitely many solutions in pairwise coprime 3 -powerful integers, i.e., positive integers $n$ for which $p \mid n$ implies $p^{3} \mid n$. This was recently proved by Nitaj who, however, was unable to verify the further conjecture that this could be done infinitely often with integers $x, y$ and $z$ none of which is a perfect cube. This is now demonstrated.
\end{abstract}

Theorem. The conjecture will follow if even one such solution can be found.

Proof. Let $a+b=c$ be one solution. Then for these values of $a, b$ and $c$ the Diophantine equation $a X^{3}+b Y^{3}=c Z^{3}$ has the solution $[1,1,1]$ in integers $[X, Y, Z]$ with $a X, b Y$ and $c Z$ pairwise coprime and hence has infinitely many. This is a special case of a well-known theorem [2], but is easily proved for our case. For starting from one such solution $[X, Y, Z]$, we find that another is given by $X^{\prime}=$ $X\left(b Y^{3}+c Z^{3}\right), Y^{\prime}=-Y\left(a X^{3}+c Z^{3}\right), Z^{\prime}=Z\left(a X^{3}-b Y^{3}\right)$, as is easily verified. Here we find that $\left(a, Y^{\prime}\right)=\left(a, Y\left(a X^{3}+c Z^{3}\right)\right)=\left(a, c Y Z^{3}\right)=1$ and so $a X^{\prime}, b Y^{\prime}$ and $c Z^{\prime}$ are pairwise coprime provided $X^{\prime}$ and $Y^{\prime}$ are; this is not always true, for we find that $\left(X^{\prime}, Y^{\prime}\right)=\left(b Y^{3}+c Z^{3}, a X^{3}+c Z^{3}\right)=\left(a X^{3}+2 b Y^{3}, 2 a X^{3}+b Y^{3}\right)=3$ or 1 according as 3 does or does not divide $a X^{3}-b Y^{3}$. However, dividing out by this common factor if it occurs, we obtain a new solution with $\left|X^{\prime} Y^{\prime} Z^{\prime}\right| \geq 2|X Y Z|$, for since the three nonzero integers $X^{\prime} / X, Y^{\prime} / Y$ and $Z^{\prime} / Z$ have sum zero, their product must be at least 2 in absolute value.

For any such solution, $x=a X^{3}, y=b Y^{3}$ and $z=c Z^{3}$ provides a solution of the original equation, and none of $x, y$ and $z$ will be a cube if none of $a, b$ and $c$ is.

The result therefore follows on observing that

$$
\begin{aligned}
& X=9712247684771506604963490444281, \\
& Y=32295800804958334401937923416351, \\
& Z=27474621855216870941749052236511,
\end{aligned}
$$

is a solution of the equation $32 X^{3}+49 Y^{3}=81 Z^{3}$, for which $7 \mid Y$.

\section{REFERENCES}

[1] P. Erdös, Problems and results on consecutive integers, Eureka 38 (1975-76), 3-8.

[2] L. J. Mordell, Diophantine equations, Academic Press, London and New York, 1969, p. 78. MR 40:2600

Received by the editor May 15, 1996 and, in revised form, September 13, 1996. 1991 Mathematics Subject Classification. Primary 11P05.

(C)1998 American Mathematical Society 
[3] A. Nitaj, On a conjecture of Erdös on 3-powerful numbers, Bull. London Math. Soc. 27 (1995), 317-318. MR 96b:11045

Department of Mathematics, Royal Holloway University of London, Egham, Surrey TW20 0EX, United Kingdom

E-mail address: J.Cohn@rhbnc.ac.uk 